\title{
Computed Tomography Findings of Pulmonary Mycobacterium simiae Infection
}

\author{
Ayeh Baghizadeh, ${ }^{1}$ Payam Mehrian, ${ }^{1}$ and Poopak Farnia ${ }^{2,3}$ \\ ${ }^{1}$ Pediatric Respiratory Diseases Research Center, National Research Institute of Tuberculosis and Lung Disease (NRITLD), \\ Shahid Beheshti University of Medical Sciences, Tehran, Iran \\ ${ }^{2}$ Mycobacteriology Research Center, National Research Institute of Tuberculosis and Lung Disease (NRITLD), \\ Shahid Beheshti University of Medical Sciences, Tehran, Iran \\ ${ }^{3}$ Department of Biotechnology, School of Advanced Technology in Medicine, \\ Shahid Beheshti University of Medical Sciences, Tehran, Iran
}

Correspondence should be addressed to Payam Mehrian; payaman2000@yahoo.com

Received 13 October 2016; Accepted 28 November 2016; Published 3 January 2017

Academic Editor: Sahal Al-Hajoj

Copyright ( 2017 Ayeh Baghizadeh et al. This is an open access article distributed under the Creative Commons Attribution License, which permits unrestricted use, distribution, and reproduction in any medium, provided the original work is properly cited.

\begin{abstract}
Nontuberculous mycobacterial (NTM) pulmonary infections can be quite similar to tuberculosis, both clinically and radiologically. However, the treatment protocol is not similar. Mycobacterium simiae is a rare cause of NTM pulmonary infection. Herein, we aimed to evaluate and compare the computed tomography (CT) scan findings of M. simiae infection in lungs. For this reason, thirty-four patients $(n=34)$ with $M$. simiae lung infection were retrospectively evaluated. Diagnosis was confirmed by American Thoracic Society (ATS) guidelines and CT scans were reviewed in both lung and mediastinal windows. The average age of patients was $63 \pm 14.54$ years and $52.9 \%$ were male. The majority of patients had cough $(91.2 \%)$ and sputum production (76.5\%). Clinically, $41.2 \%$ of patients had previous history of TB (14/34), 38.2\% had cardiac diseases (13/34), and 35.3\% had diabetes mellitus (12/34). The most common CT findings in our study were nodular lesions (100\%) and bronchiectasis (85.29\%). Regarding the severity, grade I bronchiectasis was the most prevalent. Other prominent findings were tree-in-bud sign (88.2\%), consolidation (52.94\%), and lobar fibrosis and volume loss (67.6\%). There was no significant zonal distribution of findings. In conclusion, nodular lesions and bronchiectasis are the most frequent features in CT scan of M. simiae pulmonary infection.
\end{abstract}

\section{Introduction}

Nontuberculous mycobacteria (NTM) are a large group of pathogens comprising more than 150 species and the number is continuously growing [1]. These opportunistic pathogens account for an increasing proportion of mycobacterial infections [2-4]. Reports indicate that the incidence and mortality rate of these infections have a rising trend $[5,6]$. M. simiae is one of the rare species of NTM, first described in 1965 [7]. Up to now, $M$. simiae has been reported from many regions in the world but the most prevalent regions have been Southern United States and Western Asian countries [8-10].

$M$. simiae can cause infections in various systems of body, including lungs. $M$. simiae pulmonary symptoms are nonspecific and include a combination of pulmonary and constitutional symptoms such as cough, sputum production, hemoptysis, dyspnea, fever, sweating, and weight loss [11, 12]. Underlying pulmonary diseases increase the risk of infection [12]. M. simiae infection is more prevalent in immunocompromised patients, including HIV positive patients, patients with previous history of TB, children, and the elderly [13-16]. Diabetes mellitus, cardiovascular diseases, and malignancies also increase the risk of infection [14].

Chest X-ray findings in NTM infections are usually nonspecific. These findings often do not help distinguish these infections from other conditions such as TB [17-19]. There are very few studies regarding CT scan of pulmonary M. simiae infection. Most of them have been conducted in AIDS or other immunocompromised patients and failed to reveal any specific findings $[14,20]$. In this study, we evaluated CT scan findings of pulmonary M. simiae infection. 


\section{Methods and Materials}

Between January 2011 and January 2016, 34 patients with $M$. simiae lung disease referred to Masih Daneshvari Hospital were retrospectively evaluated. In all subjects, the diagnosis of mycobacterial infection was confirmed by Masih Daneshvari Microbiology Laboratory with respect to the guidelines of the ATS, considering related symptoms, consistent radiographic abnormalities, and culture-positive sputum specimens for making a diagnosis [7]. Patients with high clinical suspicion for tuberculosis who had negative sputum cultures underwent bronchoscopy with bronchoalveolar lavage and transbronchial biopsy for a definite diagnosis. All patients underwent pulmonary CT scan. The demographic data were obtained from medical records.

CT scan was acquired with a helical technique from the lung apices to the lung bases. The images were reviewed on both lung (window width $1500 \mathrm{H}$, window level $-700 \mathrm{H}$ ) and mediastinal (window width $400 \mathrm{H}$, window level $20 \mathrm{H}$ ) windows. Right upper lobe (RUL), right lower lobe (RLL), right middle lobe (RML), left upper lobe (LUL), lingula, and left lower lobe (LLL) were reviewed for each patient, accounting for 204 lobes in total.

Statistical analysis was performed by SPSS software for Windows (version 16; IBM Corporation, Armonk, NY, USA). Qualitative analysis was conducted to figure out the frequency of CT scan findings. The results were reported in both the frequency and percentage. Zonal distribution of opacities was analyzed with $t$-test and related $p$ values were reported. The bronchiectasis severity was evaluated based on the CT scoring system for cystic fibrosis. The cases were scored from 1 to $3[21,22]$. Adenopathy station was reviewed based on the International Association for the Study of Lung Cancer (IASLC) lymph node map [23].

\section{Results}

In this study, we reviewed CT scan images of 34 patients with average age of $63 \pm 14.54$ years. 18 patients (52.9\%) were male. All patients were of Iranian origin. Habitual history included smoking in 22 patients $(64.7 \%)$, opium abuse in 10 patients (29.4\%), and alcohol abuse in two patients (5.9\%).

The presenting symptoms of lung infection were cough in 31 patients $(91.2 \%)$ and sputum production in 26 patients (76.5\%). Other symptoms included fever (18 patients, $52.9 \%$ ), dyspnea (18 patients, $52.9 \%$ ), weight loss (14 patients, $41.2 \%$ ), chest pain (13 patients, $38.2 \%$ ), and sweating (9 patients, $26.5 \%)$. Purified protein derivative (PPD) skin test for tuberculosis was positive in 9 patients (26.5\%). Underlying medical conditions and habitual history are presented in Table 1.

Lymphadenopathy, pleural effusion, and pleural thickening were seen in $15(44.11 \%), 7$ (20.6\%), and 20 (58.8\%) patients, respectively. The most common location of adenopathy was right lower paratracheal (12 patients, $35.3 \%)$, followed by right hilar (2 patients, $5.9 \%$ ) and left hilar (2 patients, 5.9\%).

Out of 204 evaluated pulmonary lobes, bronchiectasis (Figure 1) was detected in 75 lobes (36.8\%) and 29 patients $(85.29 \%)$ had bronchiectasis in at least one lobe. The most
TABLE 1: Underlying conditions in medical and habitual history (CRF: chronic renal failure, COPD: chronic obstructive pulmonary disease).

\begin{tabular}{lc}
\hline Predisposing factors in medical and habitual history & Frequency \\
\hline Smoking & $22(64.7 \%)$ \\
Opium consumption & $10(29.4 \%)$ \\
Alcohol abuse & $2(5.9 \%)$ \\
Malignancy & $1(2.9 \%)$ \\
CRF & $1(2.9 \%)$ \\
Diabetes mellitus & $12(35.3 \%)$ \\
Immunodeficiency & $1(2.9 \%)$ \\
TB history & $14(41.2 \%)$ \\
Cardiac disease & $13(38.2 \%)$ \\
Chest wall deformity & $12(35.3 \%)$ \\
COPD & $6(17.6 \%)$ \\
Cystic fibrosis & $7(20.6 \%)$ \\
\hline
\end{tabular}



Figure 1: 88-year-old male. Spiral CT scan at the level of aortic root (lung window). Cavitary nodule in right lower lobe (black arrow) along with nodular infiltration in left lower lobe, lingula, right lower lobe, and right middle lobe (white arrows). Note also cylindrical bronchiectasis in right middle lobe (thick white arrow). The hyperdense focus in superior vena cava was related to cardiac pacemaker (curved arrow).

TABLE 2: Frequency of different grades of bronchiectasis in $M$. simiae pulmonary infection.

\begin{tabular}{lcc}
\hline Bronchiectasis grade & Frequency & $p$ value \\
\hline I & $25(73.5 \%)$ & \\
II & $8(23.5 \%)$ & 0.011 \\
III & $1(2.9 \%)$ & \\
\hline
\end{tabular}

commonly involved lobe was RML (50\%), followed by lingula (47\%), RUL (41.2\%), RLL (32.4\%), LLL (32.4), and LUL (17.6\%). Most patients had mild bronchiectasis. Table 2 shows frequency of different grades of bronchiectasis in $M$. simiae pulmonary infection.

In this study, all patients had at least one nodule. We categorized nodular opacities according to the size into two groups of under and over one centimeter. Nodules under and over one centimeter were both most common in RLL 
TABLE 3: Frequency of locations of nodules under one centimeter.

\begin{tabular}{lcc}
\hline Location & Frequency & $p$ value \\
\hline Right upper lobe & $16(47.1 \%)$ & \\
Right middle lobe & $18(52.9 \%)$ & \\
Right lower lobe & $20(58.8)$ & 0.447 \\
Left upper lobe & $17(50 \%)$ & \\
Lingula & $12(35.3 \%)$ & \\
Left lower lobe & $19(55.9 \%)$ & \\
\hline
\end{tabular}

TABLE 4: Frequency of locations of nodules over one centimeter.

\begin{tabular}{lcc}
\hline Location & Frequency & $p$ value \\
\hline Right upper lobe & $5(14.7 \%)$ & \\
Right middle lobe & $7(20.6 \%)$ & \\
Right lower lobe & $9(26.5 \%)$ & 0.392 \\
Left upper lobe & $5(14.7 \%)$ & \\
Lingula & $6(17.6 \%)$ & \\
Left lower lobe & $6(17.6 \%)$ & \\
\hline
\end{tabular}

(58.8\% and $26.5 \%$, resp.) (Tables 3 and 4 ). Cavitation and airfluid level (Figure 2) were more frequent in nodules over one centimeter $(29.4 \%$ versus $8.8 \%$ for cavitation and $8.8 \%$ versus $2.9 \%$ for air-fluid level). However, calcification rate did not differ between the two groups.

Tree-in-bud opacities were observed in 30 patients (88.2\%). The distribution of tree-in-bud opacities in different lung lobes is as follows: RML (47.1\%), LLL (47.1\%), RLL (41.2\%), RUL (35.3\%), LUL (35.3\%), and lingula (32.4\%).

$7(20.58 \%)$ of our patients had masses. In 5 cases $(14.70 \%)$, the masses demonstrated cavitation. Air-fluid level was observed in 4 patients (11.76\%). Masses were most commonly located in RLL (11.8\%), RUL (8.8\%), and LLL (8.8\%).

Consolidation (Figures 2 and 3) was observed in 18 (52.94\%) cases, 3 (8.82\%) of which had cavitation (Figure 3). Consolidation was more prevalent in RUL (20.1\%), RML (11.8\%), and RLL (11.8\%).

Lobar fibrosis and volume loss were seen in 23 cases (67.6\%). The locations were RUL (55.9\%), RLL (8.8\%), LUL (5.9\%), and lingula (2.9\%). Bulla/Bleb was detected in 8 patients (23.5\%). Emphysema, chest wall deformity, and pneumothorax were seen in $14(41.2 \%), 18$ (52.9\%), and 2 (5.9\%) patients, respectively.

Although there were some differences in the distribution of CT scan findings in various lung lobes, based on $p$ values, none of these differences were statistically significant.

\section{Discussion}

There are very few studies evaluating CT scan findings in patients with $M$. simiae pulmonary infection. The patients' demographic factors and underlying medical conditions were consistent with the previous Iranian study on M. simiae pulmonary infection [11]. Maoz et al's study has also revealed that smoking, diabetes, cardiac disease, malignancy, and chronic obstructive pulmonary disease have been associated with higher incidence of $M$. simiae infection [24], although



FIGURE 2: 75-year-old male. Spiral CT scan at the level of pulmonary artery (lung window). Note consolidation with air bronchogram in lingula (black arrow) and air-fluid containing cavity in right lower lobe (thick black arrow) adjacent to pleural effusion (thick white arrow). There are also bronchiectasis in right middle lobe (curved arrow) and pneumothorax (dotted arrow).



Figure 3: 66-year-old female. Spiral CT scan below the level of left pulmonary artery (lung window). There is cavitary consolidation in posterior segment of right upper lobe (dotted arrow) along with adjacent nodular infiltration (thick arrow).

the rate of malignancy was not so much high in our study $(2.9 \%)$.

The most common findings in our study were nodular lesions (100\%) and bronchiectasis (85.29\%). Other studies have also found almost similar results. Baghaei et al. [11] have reported that patients most commonly had nodular lesions (100\%), cavitation (88.5\%), and bronchiectasis $(84.6 \%)$. Christensen et al. $[18,25]$ stated that ill-defined nodules without cavity and bronchiectasis were the most common CT findings. Consistent with our study, Song et al. [26] found bronchiectasis in $98 \%$ and cavitation in 36\% of patients. In previous studies, generally, CT scan findings were reported for NTM infections altogether rather than describing manifestations of one specific type. Shitrit et al. 
made a comparison between two species of mycobacteria and found lower rates of cavitation in M. simiae than M. kansasii (3\% versus $57 \%$, $p$ value $=0.001)$ [14]

Lymphadenopathy was found in $46.1 \%$ of patients in our study. Lee et al. have reported similar rates around 33.3\% [25]. In contrast to our findings, Banks et al. have detected lymphadenopathy in $3 \%$ of the patients $[14,27]$. Also, $20.6 \%$ of our patients had pleural effusion while in other studies this figure varied from $4.16 \%$ to $16 \%[14,25]$.

The NTM infection patterns can be quite different between immunocompetent and immunocompromised patients. Due to paucity of immunocompromised patients in our study, we can consider our patients as immunocompetent. In immunocompetent patients, two major patterns have been described for NTM infection: an upper lobe cavitary pattern and a nodular bronchiectatic pattern [28-33]. In our study, nodular bronchiectatic form without any significant zonal distribution was more common.

The previous $\mathrm{TB}$ infection rate was high in our study (41.2\%). In the available literature, it is reported that history of pulmonary diseases including TB is more common in NTM infections [34, 35]. Shitrit et al. [14] support these findings and declare that the rate of previous pulmonary disease in $M$. simiae group is higher than $M$. kansasii patients.

Rates of scar and volume loss in different studies have been reported from $11 \%$ to $36 \%[26,34]$ but this rate in our study was even higher and reached $67.6 \%$. The cause of high rate of scar and volume loss in our study is not clear and should be investigated in further researches.

Our study's main limitations were lack of second group for comparison, its retrospective type, and limited number of patients. In future studies, presence of second group (control or infections of other types) can help obtaining more solid conclusions. In conclusion, nodular lesions and bronchiectasis are the most frequent features in CT scan findings of $M$. simiae pulmonary infection.

\section{Competing Interests}

The authors declare that they have no competing interests.

\section{References}

[1] C. L. Daley and D. E. Griffith, "Pulmonary non-tuberculous mycobacterial infections," International Journal of Tuberculosis and Lung Disease, vol. 14, no. 6, pp. 665-671, 2010.

[2] S. D’Antonio, P. Rogliani, G. Paone et al., "An unusual outbreak of nontuberculous mycobacteria in hospital respiratory wards: association with nontuberculous mycobacterial colonization of hospital water supply network," International Journal of Mycobacteriology, vol. 5, no. 2, pp. 244-247, 2016.

[3] P. M. Cassidy, K. Hedberg, A. Saulson, E. McNelly, and K. L. Winthrop, "Nontuberculous mycobacterial disease prevalence and risk factors: a changing epidemiology," Clinical Infectious Diseases, vol. 49, no. 12, pp. e124-e129, 2009.

[4] S. Halstrom, P. Price, and R. Thomson, "Review: environmental mycobacteria as a cause of human infection," International Journal of Mycobacteriology, vol. 4, no. 2, pp. 81-91, 2015.
[5] M. Mirsaeidi, R. F. Machado, J. G. Garcia, D. E. Schraufnagel, and J. L. Herrmann, "Nontuberculous mycobacterial disease mortality in the United States, 1999-2010: A Population-Based Comparative Study," PLOS ONE, vol. 9, no. 3, Article ID e91879, 2014.

[6] N. Martín-Casabona, A. R. Bahrmand, J. Bennedsen et al., "Non-tuberculous mycobacteria: patterns of isolation. A multicountry retrospective survey," International Journal of Tuberculosis and Lung Disease, vol. 8, no. 10, pp. 1186-1193, 2004.

[7] D. E. Griffith, T. Aksamit, B. A. Brown-Elliott et al., "An official ATS/IDSA statement: diagnosis, treatment, and prevention of nontuberculous mycobacterial diseases," American Journal of Respiratory and Critical Care Medicine, vol. 175, no. 4, pp. 367416, 2007.

[8] S. Rahideh, Z. Derakhshaninezhad, P. Farnia et al., "Review and meta analysis of nontuberculous mycobacteria in the Middle East," International Journal of Mycobacteriology, vol. 4, supplement 1, p. 149, 2015.

[9] G. Valero, J. Peters, J. H. Jorgensen, and J. R. Graybill, “Clinical isolates of Mycobacterium simiae in San Antonio, Texas. An 11yr review," American Journal of Respiratory and Critical Care Medicine, vol. 152, no. 5, pp. 1555-1557, 1995.

[10] R. C. Bell, J. H. Higuchi, W. N. Donovan, I. Krasnow, and W. G. Johanson Jr., "Mycobacterium simiae. Clinical features and follow-up of twenty-four patients," American Review of Respiratory Disease, vol. 127, no. 1, pp. 35-38, 1983.

[11] P. Baghaei, P. Tabarsi, P. Farnia et al., "Pulmonary disease caused by Mycobacterium simiae in Iran's national referral center for tuberculosis," Journal of Infection in Developing Countries, vol. 6, no. 1, pp. 23-28, 2012.

[12] L. Barrera, D. Palmero, R. Paul, and B. Lopez, "Disease due to Mycobacterium simiae and "Mycobacterium sherrisii" in Argentina," Medicina, vol. 70, no. 4, pp. 343-346, 2010.

[13] D. R. Phillips, H. Krishnan, and J. Watson, "First UK report of successful treatment of Mycobacterium simiae and immune reconstitution inflammatory syndrome in an HIV-seropositive patient," Sexually Transmitted Infections, vol. 84, no. 4, pp. 271$272,2008$.

[14] D. Shitrit, N. Peled, J. Bishara et al., "Clinical and radiological features of Mycobacterium kansasii infection and Mycobacterium simiae infection," Respiratory Medicine, vol. 102, no. 11, pp. 1598-1603, 2008.

[15] N. C. Patel, P. K. Minifee, M. K. Dishop, and F. M. Munoz, "Mycobacterium simiae cervical lymphadenitis," The Pediatric Infectious Disease Journal, vol. 26, no. 4, pp. 362-363, 2007.

[16] B. Braun-Saro, J. Esteban, S. Jiménez, J. M. Castrillo, and M. L. Fernández-Guerrero, "Mycobacterium simiae infection in an immunocompromised patient without acquired immunodeficiency syndrome," Clinical Infectious Diseases, vol. 34, no. 5, pp. e26-e27, 2002.

[17] J. Banks, A. M. Hunter, and I. A. Campbell, "Pulmonary infection with Myocbacterium kansasii in Wales, 1970-9: review of treatment and response," Thorax, vol. 38, no. 4, pp. 271-274, 1983.

[18] E. E. Christensen, G. W. Dietz, C. H. Ahn, J. S. Chapman, R. C. Murry, and G. A. Hurst, "Radiographic manifestations of pulmonary Mycobacterium kansasii infections," American Journal of Roentgenology, vol. 131, no. 6, pp. 985-993, 1978.

[19] D. H. Anderson, P. Grech, R. H. Townshend, and A. E. Jephcott, "Pulmonary lesions due to opportunist mycobacteria: review includes 30 cases of M. Kansasii infections," Clinical Radiology, vol. 26, pp. 461-469, 1975. 
[20] Y. Lee, J. W. Song, E. J. Chae et al., "CT findings of pulmonary non-tuberculous mycobacterial infection in non-AIDS immunocompromised patients: a case-controlled comparison with immunocompetent patients," The British Journal of Radiology, vol. 86, no. 1024, 2013.

[21] M. Bhalla, N. Turcios, V. Aponte et al., "Cystic fibrosis: scoring system with thin-section CT," Radiology, vol. 179, no. 3, pp. 783788, 1991.

[22] T. H. Helbich, G. Heinz-Peer, I. Eichler et al., "Cystic fibrosis: CT assessment of lung involvement in children and adults," Radiology, vol. 213, no. 2, pp. 537-544, 1999.

[23] A. H. El-Sherief, C. T. Lau, C. C. Wu, R. L. Drake, G. F. Abbott, and T. W. Rice, "International Association for the Study of Lung Cancer (IASLC) lymph node map: radiologic review with CT illustration," RadioGraphics, vol. 34, no. 6, pp. 1680-1691, 2014.

[24] C. Maoz, D. Shitrit, Z. Samra et al., "Pulmonary Mycobacterium simiae infection: comparison with pulmonary tuberculosis," European Journal of Clinical Microbiology \& Infectious Diseases, vol. 27, no. 10, pp. 945-950, 2008.

[25] Y. Lee, J. W. Song, E. J. Chae et al., "CT findings of pulmonary non-tuberculous mycobacterial infection in non-AIDS immunocompromised patients: a case-controlled comparison with immunocompetent patients," The British Journal of Radiology, vol. 86, no. 1024, Article ID 20120209, 2013.

[26] J. W. Song, W.-J. Koh, K. S. Lee et al., "High-resolution CT findings of Mycobacterium avium-intracellulare complex pulmonary disease: correlation with pulmonary function test results," American Journal of Roentgenology, vol. 191, no. 4, p. 1070, 2008.

[27] J. Banks, A. M. Hunter, I. A. Campbell, P. A. Jenkins, and A. P. Smith, "Pulmonary infection with Mycobacterium kansasii in Wales, 1970-9: review of treatment and response," Thorax, vol. 38, no. 4, pp. 271-274, 1983.

[28] S. M. Albelda, J. A. Kern, D. L. Marinelli, and W. T. Miller, "Expanding spectrum of pulmonary disease caused by nontuberculous mycobacteria," Radiology, vol. 157, no. 2, pp. 289-296, 1985.

[29] E. Christensen, G. Dietz, C. Ahn et al., "Pulmonary manifestations of Mycobacterium intracellularis," American Journal of Roentgenology, vol. 133, no. 1, pp. 59-66, 1979.

[30] A. A. Velayati, S. Rahideh, Z. Derakhshani Nezhad, P. Farnia, and M. Mirsaeidi, "Nontuberculous mycobacteria in Middle East: current situation and future challenges," International Journal of Mycobacteriology, vol. 4, no. 1, pp. 7-17, 2015.

[31] S. M. Ellis and D. M. Hansell, "Imaging of non-tuberculous (Atypical) mycobacterial pulmonary infection," Clinical Radiology, vol. 57, no. 8, pp. 661-669, 2002.

[32] D. L. Levin, "Radiology of pulmonary Mycobacterium aviumintracellulare complex," Clinics in Chest Medicine, vol. 23, no. 3, pp. 603-612, 2002.

[33] S. Martinez, H. P. McAdams, and C. S. Batchu, "The many faces of pulmonary nontuberculous mycobacterial infection," American Journal of Roentgenology, vol. 189, no. 1, pp. 177-186, 2007.

[34] D. Han, K. S. Lee, W. J. Koh, C. A. Yi, T. S. Kim, and O. J. Kwon , "Radiographic and CT findings of nontuberculous mycobacterial pulmonary infection caused by Mycobacterium abscessus," American Journal of Roentgenology, vol. 181, no. 2, pp. 513-517, 2003.

[35] E. Wolinsky, "Nontuberculous mycobacteria and associated diseases," The American Review of Respiratory Disease, vol. 119, no. 1, pp. 107-159, 1979. 


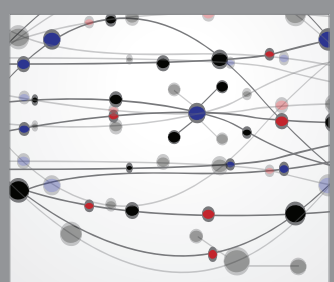

The Scientific World Journal
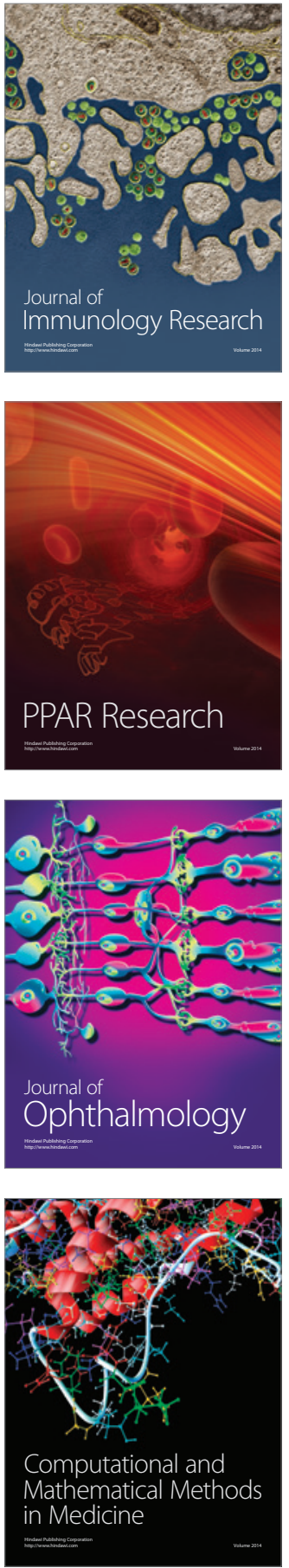

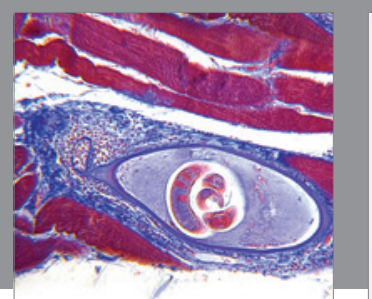

Gastroenterology Research and Practice
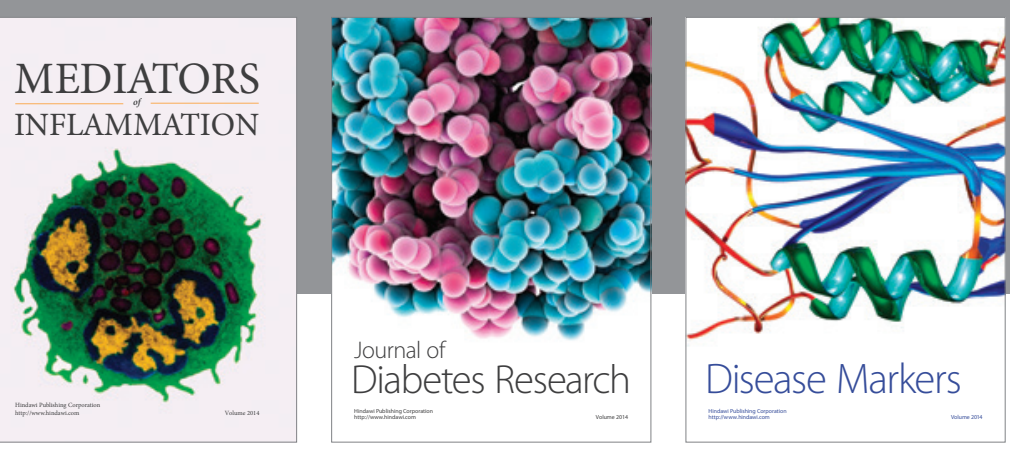

Disease Markers

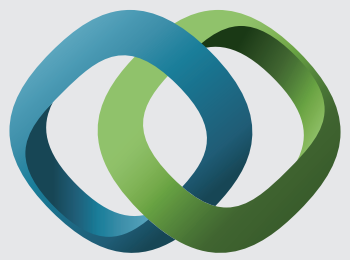

\section{Hindawi}

Submit your manuscripts at

https://www.hindawi.com
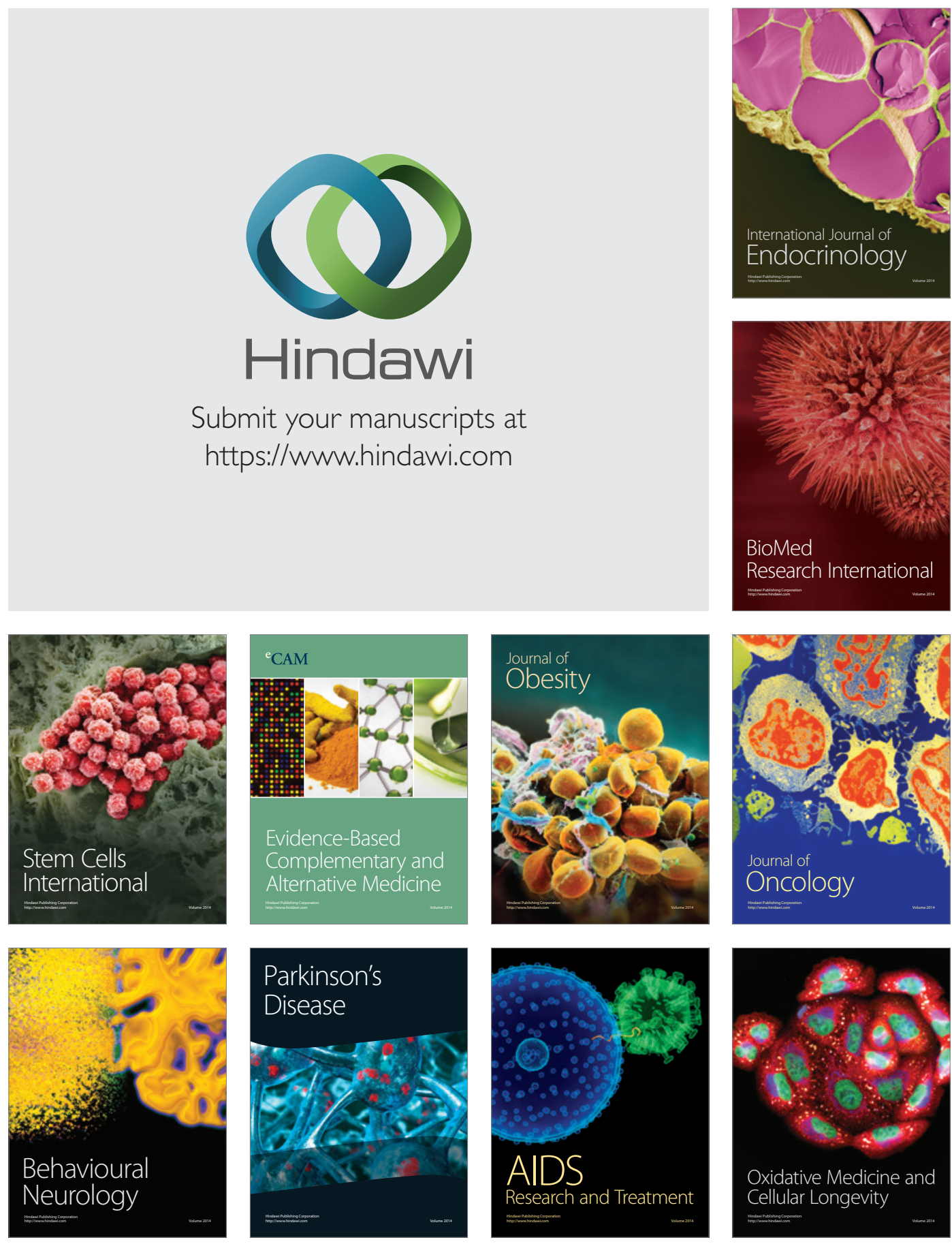